\title{
Estudos morfológicos e taxonômicos em Hypnea cornuta (Kützing) J. Agardh (Gigartinales, Rhodophyta) no litoral da Bahia, Brasil ${ }^{1}$
}

\author{
Priscila Barreto de Jesus ${ }^{2,3,5}$ e José Marcos de Castro Nunes ${ }^{2,3,4}$
}

Recebido em 9/07/2011. Aceito em 3/08/2012

\begin{abstract}
RESUMO
(Estudos morfológicos e taxonômicos em Hypnea cornuta (Kützing) J. Agardh (Gigartinales, Rhodophyta) no litoral da Bahia, Brasil). Durante estudo sobre as espécies de Hypnea do litoral do estado da Bahia foram encontrados exemplares de Hypnea cornuta (Kützing) J. Agardh em coletas realizadas no extremo sul do estado. Este é o primeiro relato de H. cornuta para o estado da Bahia, Brasil. É fornecida uma caracterização detalhada dos aspectos morfológicos, anatômicos e reprodutivos desta espécie.
\end{abstract}

Palavras-chave: Bahia, nova ocorrência, morfologia, Rhodophyta, taxonomia

\begin{abstract}
(Morphological and taxonomic studies in Hypnea cornuta (Kützing) J. Agardh (Gigartinales, Rhodophyta) in the coast of Bahia, Brazil). During study of the species of Hypnea of the coast of Bahia specimens of Hypnea cornuta (Kützing) J. Agardh were found in the southern state. This is the first report of H. cornuta for the state of Bahia, Brazil. It is provided a detailed characterization of morphological, anatomical and reproductive aspects of this species.
\end{abstract}

Key words: Bahia, new occurrence, morphology, Rhodophyta, taxonomy

\section{Introdução}

O gênero Hypnea J.V. Lamouroux compreende 67 espécies (Guiry \& Guiry 2012) amplamente distribuídas nos mares tropicais e subtropicais de todo o mundo, estando presente em praticamente todas as listas de floras de algas bentônicas dos Oceanos Atlântico, Índico e Pacífico (Mshigeni \& Chapman 1994). Wynne (2011) reconhece para a costa tropical e subtropical da América nove espécies: $H$. cenomyce J. Agardh, H. cervicornis J. Agardh, H. cornuta (Kützing) J. Agardh, H. krugiana Hauck, H. musciformis (Wulfen in Jacq.) J.V. Lamour., H. nigrescens Grev. ex J. Agardh, H. spinella (C. Agardh) Kütz, H. valentiae (Turner) Mont. e H. volubilis Searles. Destas, apenas H. krugiana não é citada para o Brasil (Creed et al. 2012).

Hypnea cornuta foi originalmente descrita para o litoral africano, leste do Oceano Atlântico (Kützing 1849, como Chondroclonium cornutum; J. Agardh 1852, como
H. cornuta var. cornuta); entretanto, tem sido citada com frequência apenas para a costa do Oceano Pacífico (Bangmei \& Yongquiang 1997, Lewmanomont 1997; Chiang 1997) e do Índico (Silva et al. 1996; Lipkin \& Silva 2002).

No litoral brasileiro a citação desta espécie é escassa e restrita a trabalhos realizados antes da década de 90 , não havendo relatos em coletas recentes. Até o momento, a única referência de Hypnea cornuta no Brasil efetivamente publicada foi feita por Joly et al. (1968) para o estado do Rio de Janeiro. Os demais estudos que fazem referência a este táxon no país encontram-se sob a forma de Teses ou Dissertações (Yoneshigue-Valentin 1985, Schenkman 1986 e Reis-Santos 1990), o que dificulta sua localização e consulta. Além disso, os referidos trabalhos não definem a procedência exata do material ou não apresentam descrições ou ilustrações detalhadas.

A partir do estudo das espécies do gênero Hypnea ocorrentes no litoral do Estado da Bahia, foram encontrados

\footnotetext{
${ }^{1}$ Parte da dissertação de Mestrado da primeira Autora.

${ }^{2}$ Universidade Estadual de Feira de Santana, Programa de Pós-graduação em Botânica, Departamento de Ciências Biológicas, Feira de Santana, BA, Brasil

${ }^{3}$ Universidade Federal da Bahia, Departamento de Botânica, Instituto de Biologia, Laboratório de Algas Marinhas, Salvador, BA, Brasil

${ }^{4}$ Universidade do Estado da Bahia, Departamento de Ciências Exatas e da Terra, Campus II, Alagoinhas, BA, Brasil

${ }^{5}$ Autor para correspondência: priscilla_b.j@hotmail.com
} 
espécimes típicos de $H$. cornuta, apresentando talo com abundantes e notórios processos estrelados (propágulos).

O presente trabalho tem por objetivo descrever os aspectos morfológicos, anatômicos e reprodutivos dos exemplares de Hypnea cornuta. Este estudo constitui a primeira referência desta espécie para estado da Bahia, ainda sem descrição e ilustração detalhada para o litoral do Brasil.

\section{Material e métodos}

O material proveniente do litoral baiano foi coletado no extremo sul, no município de Santa Cruz de Cabrália,

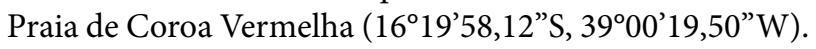
As coletas foram realizadas no mediolitoral, em substrato arenoso, na região protegida do recife e em poças, nas marés baixas de sizígia.

Os exemplares foram fixados em formol a $4 \%$, diluído em água do mar. Cortes histológicos à mão livre foram realizados com auxílio de lâminas de barbear e corados com solução de azul de anilina a 5\%. A identificação foi feita em estereomicroscópio (Leica ${ }^{\circledR}$ ZOOM 2000) e microscópio fotônico (Olympus $\left.{ }^{\oplus} \mathrm{CBA}\right)$, com ocular de medição acoplada, observando-se a morfologia externa e interna das estruturas vegetativas.

Fotomicrografias das estruturas foram obtidas com o auxílio de programa de captura de imagem (QCapture Pro) e câmera digital (QImaging GO-3) acoplada ao fotomicroscópio (Olympus trinocular CX31RTS5). O material identificado foi registrado e depositado no Herbário Alexandre Leal Costa (ALCB) do Departamento de Botânica do Instituto de Biologia da Universidade Federal da Bahia.

\section{Resultados e discussão}

Hypnea cornuta (Kütz.) J. Agardh, Sp. Gen. Ord. Alg. 1852 (2): 449.

Chondroclonium cornutum Kützing, Spec. Alg. 1849: 741. Localidade tipo: "ad oras Guineae" (J. Agardh, 1852: 449)

Figuras: 1-14

Talo ereto abundantemente ramificado, fracamente emaranhado na região basal, de textura cartilaginosa e coloração amarela amarronzada, com até $15 \mathrm{~cm}$ de altura. Fixo ao substrato através de ramos rizoidais $(0,5-1,5 \mathrm{~cm})$, pouco ou não ramificados, apresentando discos de fixação primários e secundários. Eixo principal cilíndrico e evidente, cujo diâmetro diminui gradativamente da base $(937-1.350 \mu \mathrm{m})$ em direção ao ápice (371-435 $\mu \mathrm{m})$, que se apresenta ereto e agudo terminando em uma nítida célula apical. Ramos superiores livres com 3-6,5 cm de comprimento, inseridos de maneira irregular em vários planos, ramificação em ângulos de $60-80^{\circ}$ até a segunda ordem. Râmulos espinescentes com 0,1-0,7 cm de comprimento, dispostos de maneira alterna com tendência dística. Processos estrelados peltados, com 3 a 6 pontas, frequentes na porção superior dos ramos se- cundários e râmulos, de tamanho variável (712-2.156 $\mu \mathrm{m})$ e tonalidade mais escura; apresentam constrição em sua base que funciona como zona de abscisão, desprendendo-se facilmente da planta. Alguns processos estrelados com região apical modificada formada por conjunto de células apicais rizoidais proeminentes e de parede espessa. Talo em corte transversal com uma célula axial de contorno circular, com 29-81 $\mu \mathrm{m}$ de diâmetro, muito menor que as periaxiais, as quais são ovais, em número de cinco, medindo de 98 - $229 \mu \mathrm{m} ; 2-3$ camadas de células medulares hialinas (32-80 $\mu \mathrm{m})$ gradualmente menores em direção à periferia; espessamentos lenticulares abundantes com até $57 \mu \mathrm{m}$ de comprimento nas paredes das células periaxiais e/ou medulares, (1)-2 camadas de células corticais 17-22 $\mu \mathrm{m}$ pigmentadas que, em vista superficial, apresentam-se isodiamétricas com 16-20 $\mu \mathrm{m}$ de diâmetro. Cutícula muito espessa em volta de todo o talo com 29-57 $\mu \mathrm{m}$ em corte transversal e até $116 \mu \mathrm{m}$ em vista superficial. Soros tetrasporangiais circundando a porção basal e mediana dos râmulos, 843-1.912 $\mu \mathrm{m}$ de comprimento e 350-436 $\mu \mathrm{m}$ de diâmetro, geralmente formados na região inferior do talo. Tetrasporângios divididos zonadamente, medindo $38-63 \mu \mathrm{m}$ de comprimento. Gametófitos não observados.

Material examinado: BRASIL. Bahia: Santa Cruz de Cabrália, Coroa Vermelha, 08/IX/2010, P.B. Jesus, G.M. Lyra \& J.M.de C. Nunes (ALCB).

Material adicional examinado: BRASIL. Maranhão: Recife do Timbuba, 03/XI/1982, M.M. Ferreira-Correia (SP). Rio de Janeiro: Armação de Búzios, Ponta do Pai Vitório, 28/I/1981, Y. Yoneshigue-Valentin, (RFA); Búzios, Ponta do Lagoinha, 19/II/1981, Y. Yoneshigue-Valentin (RFA); Cabo Frio, s/loc., 23/V/1976, C. Nassar (RFA); Canal de Itajurú, 17/II/1986, R.P. Reis-Santos et al. (RB); Ilha do Japonês, 27/V/1987, R.P. Reis-Santos (RB); Ponta do Ambrósio, 2/IX/1986, R.P. Reis-Santos (RB); Ponta do Costa, 12/ XII/1986, R.P. Reis-Santos (RB); Praia do Forte São Mateus, 25/II/1963, Y. Yoneshigue-Valentin (RFA); Saco do Sorita, 8/IX/1987, R.P. Reis-Santos (RB); Lagoa de Araruama, Praia dos Coqueiros, 18/II/1984, Y. Yoneshigue-Valentin (RFA); Paraty, Ilha Comprida, 25/XI/1985, M.A.O. Figueiredo (RB); São Gonçalo, Ilha Jurubaíba, 24/V/1968, Y. Yoneshigue-Valentin (RFA). São Paulo: São Sebastião, Praia do Araçá, 29/IX/1983, R.P.F Schenkman (SPF - como H. valentiae). Paraná: Ilha do Mel, Saco do Limoeiro, 4/XII/1983, E.C. Oliveira \& E. Plastino (SPF - como H. valentiae).

Os exemplares foram encontrados crescendo no mediolitoral, recobertos por substrato arenoso, em locais protegidos da arrebentação das ondas, ocorrendo solitários ou agregados, geralmente associados à Hypnea cervicornis. Servindo de hospedeiro para numerosos talos de cianofíceas.

Os espécimes estudados foram identificados como Hypnea cornuta devido à presença de fronde alongada com esparsos râmulos estrelados (Fig. 2), características diagnósticas descritas por Agardh (1852) para definir esta espécie. Os râmulos estrelados funcionam como propágulos 
(Yamagishi et al. 2003, Cecere et al. 2004; 2011) e constituem uma importante característica taxonômica para identificação desta espécie (Agardh 1852, Masuda et al. 1997, Cecere et al. 2004; 2011).

O material analisado concorda com as descrições e ilustrações fornecidas por Tanaka (1941), Taylor (1960), Mshigeni \& Chapman (1994), Wynne (1995), Chiang (1997), Cecere et al. (2004) e Dawes \& Mathielson (2008).

O status de Hypnea cornuta tem sido debatido por alguns autores. BØrgesen (1943) apontou que $H$. cornuta e $H$. valentiae eram formas bem relacionadas de uma mesma espécie, cujas diferenças eram devido às variações de condições ambientais. Esta posição foi suportada por Wynne $(1998,2005)$ em seus catálogos da costa do Atlântico tropical americano e seguida por outros autores em floras e catálogos anteriores, a exemplo de Lawson \& John (1987) e Schneider \& Searles (1991). Entretanto, os comentários sobre a possível coespecificidade fornecidos por BØrgesen (1943) foram desconsiderados por Tanaka (1941), Taylor (1960), Mshigeni \& Chapman (1994), Chiang (1997), Yamagishi \& Masuda (1997) e Dawes \& Mathielson (2008), que continuaram a reconhecer $H$. cornuta.

Segundo Abbott (1999) grande parte dos espécimes analisados de Hypnea valentiae do Havaí apresentam apenas râmulos simples e espinescentes, considerando a presença de râmulos estrelados uma característica pouco confiável para H. valentiae. Mshigeni \& Chapman (1994) afirmam que $H$. valentiae é distinta de $H$. cornuta pela presença de râmulos horizontais, dispersos, subulados, pequenos e geralmente simples como foi exposto em sua descrição original (Turner 1809, como Fucus valentiae Turner). Yamagishi et al. (2003) ressaltam que a produção de râmulos estrelados que funcionam como órgãos de propagação é uma característica única de H. cornuta. Recentemente Wynne (2011) passou a considerar $H$. cornuta como uma espécie independente de $H$. valentiae baseado nas diferenças morfológicas apontadas por Mshigeni \& Chapman (1994) e Yamaguishi et al. (2003) para distinguir estas duas espécies (Tab. 1).

Duas variedades de Hypnea cornuta foram distinguidas por Agardh (1852) em seu arranjo sistemático: var. cornuta, apresentando fronde alongada e delgada, com esparsos râmulos estrelados e, var. stellulifera, exibindo um talo mais rígido e com densos râmulos estrelados. Com base nas diferenças morfológicas apontadas e suportados pela análise de sequências do gene plastidial que codifica a subunidade larga da rubisco ( $r b c \mathrm{~L})$, Yamagish et al. (2003) elevaram $H$. cornuta var. stellullifera à categoria de espécie: $H$. stellulifera (J. Agardh) Yamagishi et Masuda, sendo esta, endêmica da Ásia tropical (Yamagish et al. 2003).

Além dos típicos processos estrelados, a presença de uma cutícula espessa transparente (Joly et al. 1968, Cecere et al. 2004) evidente tanto em corte transversal (Fig. 10) quanto em vista superficial (Fig. 12) auxilia a diferenciar Hypnea cornuta das demais espécies do gênero. No entanto Taylor (1960) ressalta que, quando os râmulos estrelados são escassos, pode ser difícil distinguir esta espécie de H. cervicornis. Os exemplares do litoral baiano foram encontrados crescendo junto a espécimes de H. cervicornis, apresentando mesma coloração e, portanto, podendo ser confundidos em campo. A presença dos râmulos estrelados em $H$. cornuta e o padrão de ramificação foram considerados características essenciais para a identificação dos exemplares (Tab. 1).

Cecere et al. (2011) caracterizaram os râmulos estrelados de Hypnea cornuta como propágulos multicelulares, apresentando características adaptativas tais como a presença de uma constrição basal que atua como zona de abscisão (Fig. 5), a capacidade de formar outros propágulos ao seu redor e a possibilidade de germinação in situ. Dentre os exemplares examinados foram encontrados alguns portando râmulos estrelados com ápices modificados (Fig. 6), cujas células apicais são proeminentes e apresentam uma espessa

Tabela 1. Comparativo das características diagnósticas entre espécies morfologicamente similares à Hypnea cornuta. $1=$ Tanaka (1941), 2 = Agardh (1852), 3= Schenkman (1986), 4= Lawson \& John (1987), 5 = Chiang (1997), 6= Yamagishi \& Masuda (1997), 7 = Abbot (1999), e 8= Dawes \& Mathielson (2008).

\begin{tabular}{|c|c|c|c|c|}
\hline & H. cornuta ${ }^{1,2,3,5,6}$ & H. cervicornis s, $^{1,3,4,5,6,7}$ & H. valentiae ${ }^{2,3,4,5,6,7}$ & Material estudado \\
\hline Tamanho $(\mathrm{cm})$ & $4-40$ & $7-30$ & $6-50$ & $7-12$ \\
\hline Cor & $\begin{array}{l}\text { Parda-amarelada, } \\
\text { esverdeada ou avermelhada }\end{array}$ & $\begin{array}{c}\text { Amarelo-esverdeado } \\
\text { ou avermelhado }\end{array}$ & Róseo avermelhada & Amarela amarronzada \\
\hline Textura & $\begin{array}{c}\text { Membranácea a } \\
\text { cartilaginosa }\end{array}$ & $\begin{array}{c}\text { Membranácea a } \\
\text { cartilaginosa }\end{array}$ & Membranácea & Cartilaginosa \\
\hline Tipo de fixação & $\begin{array}{c}\text { Células rizoidais e discos } \\
\text { de fixação }\end{array}$ & $\begin{array}{l}\text { Estolão com discos de } \\
\text { fixação }\end{array}$ & Disco de fixação & $\begin{array}{l}\text { Rizóides e discos de } \\
\text { fixação }\end{array}$ \\
\hline Padrão de ramificação & Alterna a dística & Divaricato a subdicotômica & Espiralada a alterna & Alterna a dística \\
\hline Râmulos estrelados & Presente & Ausente & Ausente & Presente \\
\hline Tipo de Ápice & Agudo & Agudo ou bifurcado & Agudo & Agudo \\
\hline Número de células periaxiais & $4-7$ & $4-6$ & $5-7$ & $5-6$ \\
\hline Espessamento lenticular & Ausente & Ausente & Raro & Presente \\
\hline Posição dos soros tetrasporangiais & Basal ou mediano & Basal & Basal ou mediano & Basal ou mediano \\
\hline
\end{tabular}



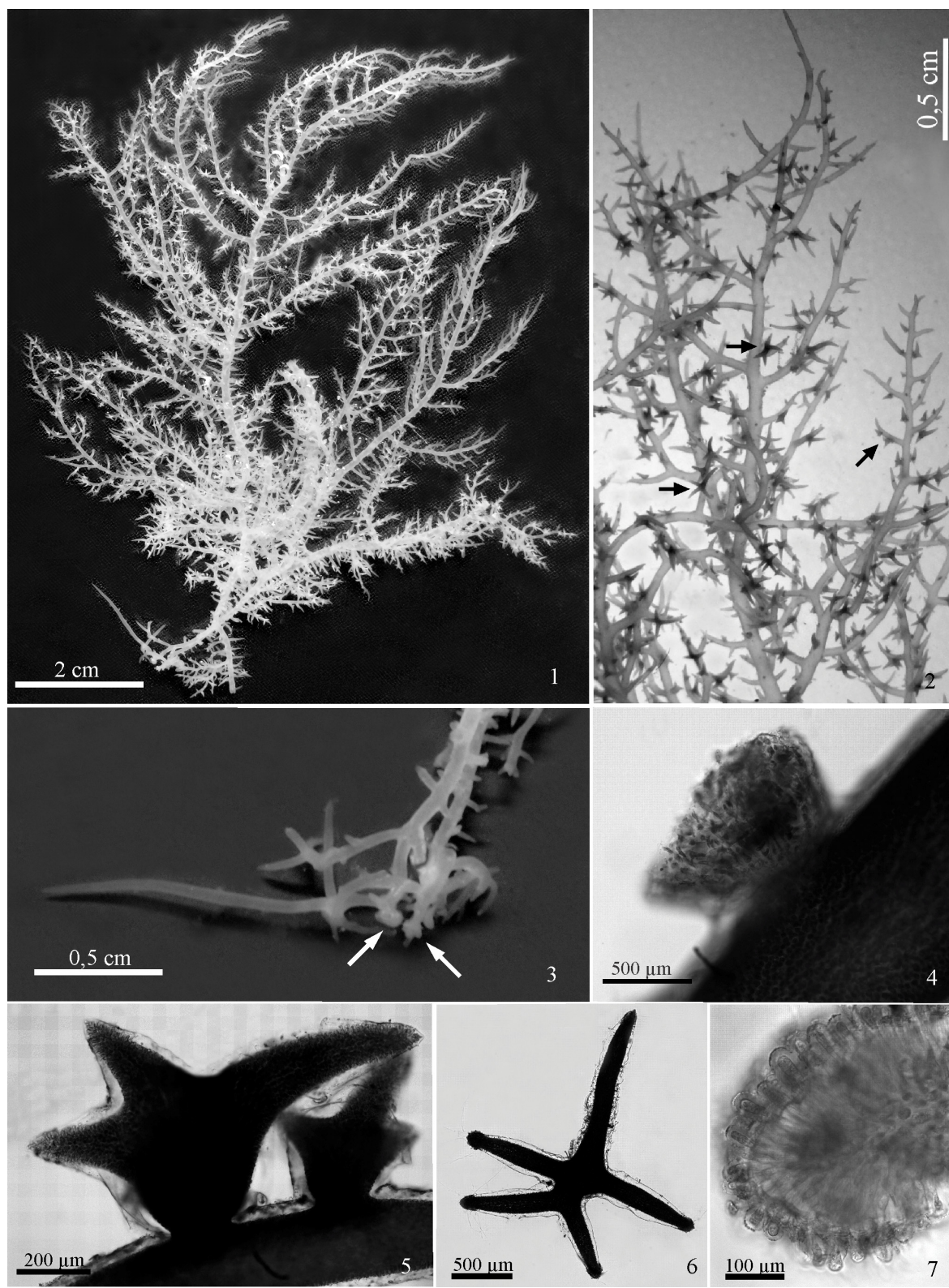

Figuras 1-7. Aspectos morfológicos de Hypnea cornuta (Kützing) J. Agardh. 1 - Aspecto geral; 2 - Detalhe do talo portando râmulos estrelados (setas); 3 - Rizóides com discos de fixação secundários (setas); 4 - Disco de fixação em detalhe; 5 - Râmulos estrelados peltados; 6 - Râmulo estrelado com ápices modificados em estágio inicial de desenvolvimento do sistema de fixação; 7 - Detalhe do ápice modificado dos râmulos estrelados com células apicais rizoidais proeminentes e parede celular espessa.

parede celular (Fig. 7). Estas características concordam com as apontadas por Cecere et al. (2004) para descrever o estágio inicial de desenvolvimento de um disco de fixação à partir de células rizoidais. Cecere et al. (2011) ressaltam também que os rizóides podem estar presentes mesmo antes da abscisão. Desta maneira, pode-se sugerir que no material analisado, os ápices modificados dos râmulos estrelados encontram-se em fase inicial de desenvolvimento de um sistema de fixação.

A maioria dos estudos relata a ausência de exemplares férteis de Hypnea cornuta sendo a reprodução exclusiva- mente vegetativa realizada através de propágulos, como observado por Tanaka (1941) para o Japão, Wynne (1995) para as ilhas de Seychelles, Chiang (1997) para Taiwan e Cecere et al. (2004) para o mediterrâneo. Taylor (1960) cita a presença de cistocarpos em exemplares americanos sem, no entanto, definir a origem do material. Lipkin \& Silva (2002) e Dawes \& Mathielson (2008) relatam a presença de tetrasporângios e cistocarpos para o material proveniente do Mar Vermelho e Flórida respectivamente. Neste estudo foram encontrados apenas exemplares tetraspóricos (Fig. 13). A presença de tetrasporângios também foi relatada por 


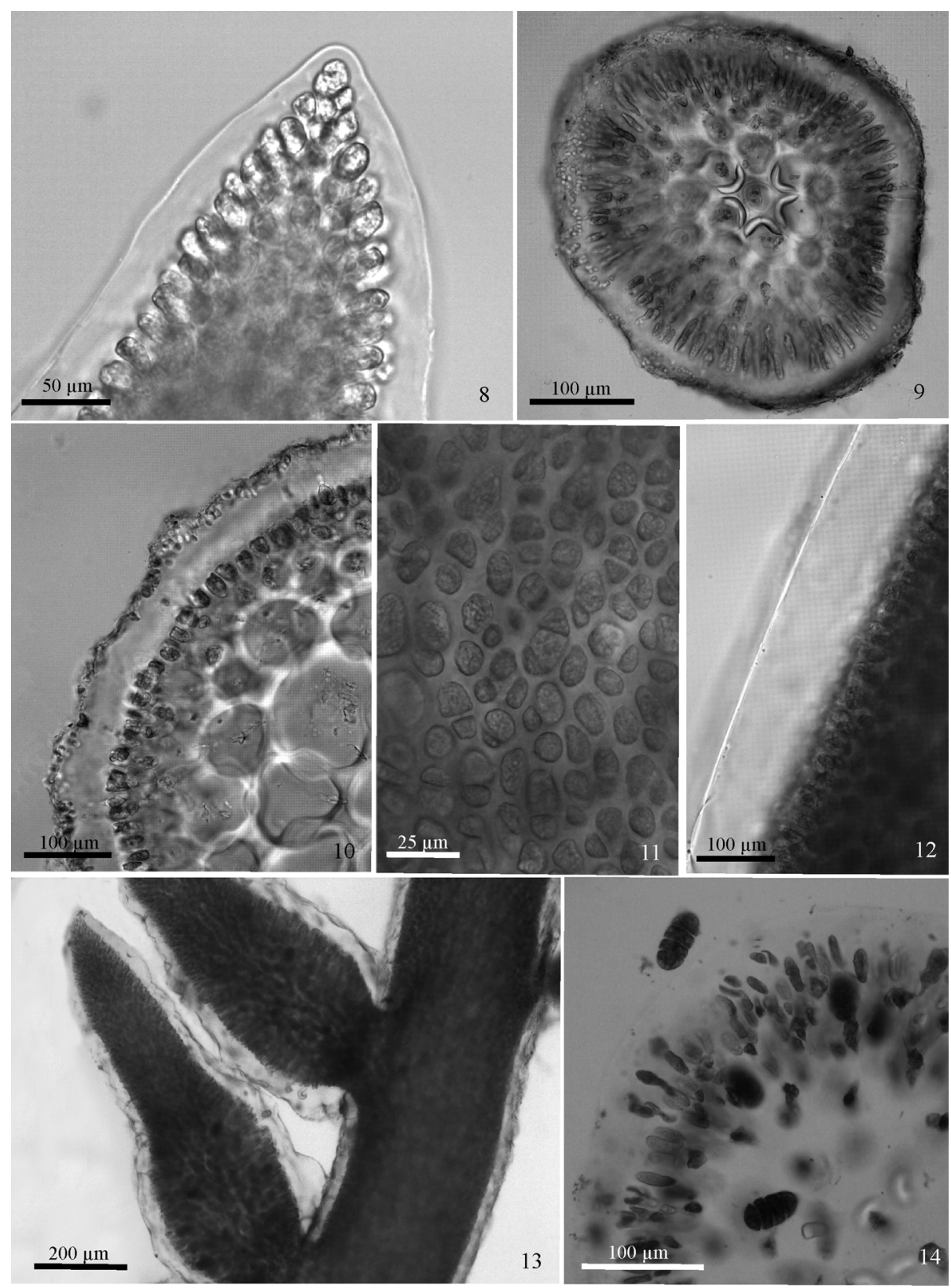

Figuras 8-14. Aspectos morfológicos e reprodutivos de Hypnea cornuta (Kützing) J. Agardh. 8 - Célula apical de um ramo secundário; 9 - Corte transversal na base de um râmulo fértil com espessamentos lenticulares nas paredes das células periaxiais; 10 - Corte transversal a $3 \mathrm{~mm}$ do ápice evidenciando as células corticais e a cutícula espessa; 11 - Células corticais em vista superficial; 12: Vista superficial da cutícula; 13 - Râmulos férteis dilatados na base após a diferenciação dos tetrasporângios (estiquídios); 14 - Corte transversal do estiquídio com tetrasporângios zonados na região cortical.

Ogawa \& Lewmanomont (1981) na Tailândia e Bangmei \& Yongqiang (1997) na China.

Embora este táxon não seja referido nos trabalhos atuais no litoral do Brasil, é possível que apresente maior área de ocorrência, como já foi verificado em estudos anteriores. Schenkman (1986) refere essa espécie para as localidades de Praia do Araçá, Praia do Arrastão e Praia do Segredo (São Sebastião), estado de São Paulo; Cabo Branco (João Pessoa), estado da Paraíba; Praia de Boa Viagem e Ponta das Pedras
(Recife), estado de Pernambuco; Vila de Peroba (Maragogi) e Praia de Ponta Verde (Maceió), estado de Alagoas. Entretanto, apesar da aparente ampla distribuição no nordeste do Brasil, durante a revisão dos herbários brasileiros, apenas a exsicata referente ao estado do Maranhão (SP 187768) foi encontrada, devendo ser confirmadas as citações para os outros estados nordestinos.

A análise de espécimes depositados em herbários brasileiros comprovou ampla distribuição de Hypnea cornuta no 
estado do Rio de Janeiro, principalmente no município de Cabo Frio. Entretanto, é digno de ressalva que todas as exsicatas analisadas datam de coletas antigas, sendo a mais recente de 1987. A confusão na identificação destes espécimes, na ausência dos râmulos estrelados, pela similaridade com outras espécies e a carência de taxonomistas no Brasil, podem ser os fatores que contribuem para esta rara distribuição.

Além dos estados da Bahia e do Maranhão no nordeste do Brasil, a partir deste estudo foi confirmada e ampliada a área de ocorrência de Hypnea cornuta no litoral brasileiro para os estados de São Paulo e Paraná, uma vez que esta era efetivamente referida apenas para o Rio de Janeiro (Joly et al. 1968 e Creed et al. 2012).

\section{Agradecimentos}

À CAPES, pela bolsa de estudos concedida à primeira autora. Ao Programa de Pós-Graduação em Botânica da Universidade Estadual de Feira de Santana, pelo financiamento para as coletas de campo. Ao Laboratório de Algas Marinhas do Instituto de Biologia da Universidade Federal da Bahia, pela disponibilização da infraestrutura utilizada.

\section{Referências bibliográficas}

Abbott, I.A. 1999. Marine red algae of the Hawaiian Islands. Bishop Museum Press, Honolulu, Hawaii.

Agardh, J.G. 1852. Species genera et ordines algarum. Vol 2. Pars 2. Gleerup, Lund.

Bangmei, X. \& Yongqiang, W. 1997. Some species of the genus Hypnea (Gigartinales, Rhodophyta) from China. Pp.193-206. In: (Abbott, I.A. Ed.) Taxonomy of economic seaweeds, Vol. VI. La Jolla, California Sea Grant College, University of California.

BØrgesen, F. 1943. Some marine algae from Mauritius. III. Rhodophyceae. Part 2. Gelidiales, Cryptonemiales, Gigartinales. Kongelige Danske Videnskabernes Selskab, Biologiske Meddelelser 19(1): 85.

Cecere, E.; Petrocelli, A. \& Verlaque, M. 2004. Morphology and vegetative reproduction of the introduced species Hypnea cornuta (Rhodophyta, Gigartinales) in the Mar Piccolo of Tarant (Italy), Mediterranean Sea. Botanica Marina 47: 381-388.

Cecere, E.; Petrocelli, A. \& Verlaque, M. 2011. Vegetative reproduction by multicellular propagules in Rhodophyta: an overview. Marine Ecology 1-19.

Chiang, Y.M. 1997. Species de Hypnea Lamouroux (Gigartinales, Rhodophyta) from Taiwan. Pp. 163-177. In: Abbott, I.A. (Ed.). Taxonomy of economic seaweeds, Vol. VI. La Jolla, California Sea Grant College, University of California.

Creed, M.; Nunes, J.M.C.; Moura, C.W.N.; Fujii, M.T.; Cassano, V.; BarrosBarreto, M.B.B. de; Pereira, S.M.B.; Khader, S.; Necchi Jr., O.; Oliveira, M.C.; Henriques, M.C.; Oliveira-Carvalho, M.F.; Guimarães, S.M.P.B. 2012. Rhodophyceae. In: Lista de Espécies da Flora do Brasil. Jardim Botânico do Rio de Janeiro. http://floradobrasil.jbrj. gov.br/2012/FB100399. (Acesso em 26/07/2012).

Dawes, C.J. \& Mathielson, A.C. 2008. The seaweeds of Florida. University Press of Florida.

Guiry, M.D. \& Guiry, G.M. 2012. AlgaeBase. World-wide electronic publication, National University of Ireland, Galway. http://www. algaebase.org. (Acesso em 26/07/2012).
Joly, A.B.; Ferreira, M.M.; Pinheiro-Vieira, F. \& Yoneshigue-Braga, Y. 1968. Additions to the American South Atlantic Marine Algae. I. Arquivos da Estação de Biologia Marinha da Universidade Federal do Ceará 8(2):177-181.

Kützing, F.T. 1849. Species algarum. Brockhaus, Lipsiae [Leipzig].

Lawson, G.W. \& John, D.M. 1987. The marine algae and coastal environment of tropical West Africa (2nd ed.) Beih. Nova Hedwigia 93: 1-415

Lewmanomont, K. 1997. Species of Hypnea from Thailand. Pp. 179-191. In: Abbott, I.A. (Ed.) Taxonomy of economic seaweeds. Vol. VI. California Sea Grant College, University of California, La Jolla.

Lipkin, Y. \& Silva, P.C. 2002. Marine algae and seagrasses of the Dahlak Archipelago, southern Red Sea. Nova Hedwigia 75: 1-90.

Masuda, M.; Yamagishi, Y.; Chiang, Y.-M., Lewmanomont, K. \& Xia, B.M. 1997. Overview of Hypnea (Rhodophyta, Hypneaceae). Pp. 127-133. In: Abbott, I.A. (Ed.) Taxonomy of economic seaweeds. Vol. VI. La Jolla, California Sea Grant College, University of California.

Mshigeni, K. E. \& Chapman, D. J. 1994. Hypnea (Gigartinales,Rhodophyta). Pp. 245-281. In: Akatsuka, I. (Ed.) Biology of Economic Algae. The Hague, SPB Academic Publishing.

Nunes, J.M.C. 2005. Rodofíceas marinhas bentônicas do estado da Bahia, Brasil. Tese de Doutorado. Instituto de Biociências da Universidade de São Paulo. Departamento de Botânica, São Paulo.

Ogawa, H. \& Lewmanomont, K. 1981. Economic seaweeds of Thailand. I. The genus Hypnea in the Vicinity of Si Racha, Chonburi Province. Kasetsart University Fisheries Research Bulletin 12: 1-14.

Reis-Santos, R.P. 1990. Flora algal da Lagoa de Araruama, Rio de Janeiro. Dissertação de Mestrado. Departamento de Ciências Biológicas da Universidade Federal do Rio de Janeiro, Rio de Janeiro.

Schenkman, R.P.F. 1986. Cultura de Hypnea (Rhodophyta) in vitro como subsídio para estudos morfológicos, reprodutivos e taxonômicos. Tese de Doutorado. Instituto de Biociências da Universidade de São Paulo, São Paulo.

Schneider, C.W. \& Searles, R.B. 1991. Seaweeds of the Southeastern United States. Durham \& London, Duke University Press.

Silva, P.C., Basson, P.W. \& Moe, R.L. 1996. Catalogue of the marine algae of the Indian Ocean. University of California Publications in Botany 79: 1-1259.

Tanaka, T. 1941. The genus Hypnea from Japan. Scientifc Papers of the Institute of Algological Research, Faculty of Science, Hokkaido Imperial University 2: 227-250.

Taylor, W.R., 1960. Marine algae of the eastern tropical and subtropical coasts of the Americas. Ann Arbor, University of Michigan Press.

Turner, D. 1809. Fuci. Vol II. Londres, John \& Arthur Arch, Cornhill.

Wynne, M.J. 1995. Benthic marine algae from the Seychelles collected during the R/V Te Vega Indian Ocean expedition. University of Michigan Herbarium 20(2): 261-346.

Wynne, M. J. 1998. A checklist of the benthic marine algae of the tropi$\mathrm{cal}$ and subtropical western Atlantic: first revision. Nova Hedwigia, Beiheft, 16 (10): 1-155.

Wynne, M. J. 2005. A checklist of benthic marine algae of the tropical and subtropical western Atlantic: second revision. Nova Hedwigia, Beiheft, 129: 1-152.

Wynne, M. J. 2011. A checklist of the benthic marine algae of the tropical and subtropical Western Atlantic: third revision. Nova Hedwigia, Beiheft, 140: 1-160.

Yamagishi, Y. \& Masuda, M. 1997. Species de Hypnea from Japan. Pp.: 135-162. In: Abbott, I.A. (Ed.) Taxonomy of economic seaweeds. Vol. VI. California Sea Grant College, University of California, La Jolla.

Yamagishi, Y., Masuda, M., Abe, T., Uwai, S., Kogame, K., Kawaguchi, S., Phang, S.M. 2003. Taxonomic notes on marine algae from Malaysia. XI. Four species of Rhodophyceae. Botanica Marina 46: 534-547.

Yoneshigue, Y. 1985. Taxonomie et ecologie des algues marines dans la region de Cabo Frio (Rio de Janeiro, Bresil). Tese de Doutorado. Faculté dês Sciences de Lumin. L'Universite D’aix - Marseille I. 